\title{
Preoperative charcoal suspension tattoo for the detection of differentiated thyroid cancer recurrence
}

\author{
FRANCESCO SOPRANI ${ }^{1}$, ANDREA DE VITO ${ }^{2}$, FABIO BONDI $^{3}$, GIUSEPPE MAGLIULO ${ }^{4}$, \\ SOON SUE RENE ${ }^{5}$, CATERINA CAPPI ${ }^{3}$, FABRIZIO RIGANTI ${ }^{6}$, CLAUDIO VICINI ${ }^{2,7}$ and MAURIZIO PUCCETTI ${ }^{6}$ \\ ${ }^{1}$ Head and Neck Department, ENT Unit, Santa Maria delle Croci Hospital, Ravenna-AUSL of Romagna, \\ I-48121 Ravenna; ${ }^{2} \mathrm{Head}$ and Neck Department, ENT and Oral Surgery Unit, Morgagni-Pierantoni Hospital, \\ Forlì-and-Santa Maria delle Croci Hospital, Ravenna AUSL of Romagna, I-47121 Forlì; ${ }^{3}$ Department of Endocrinology, \\ Santa Maria delle Croci Hospital, AUSL of Romagna, I-48121 Ravenna; ${ }^{4}$ Department of 'Organi di Senso', \\ University 'Sapienza', I-00161 Rome, Italy; ${ }^{5}$ Department of Otolaryngology Head and Neck Surgery, \\ Ng Teng Fong General Hospital, Jurong Community Hospital, National University Health System, Singapore 648346, \\ Republic of Singapore; ${ }^{6}$ Department of Pathology, Santa Maria delle Croci Hospital, AUSL of Romagna, \\ I-48121 Ravenna; ${ }^{7}$ Department ENT and Audiology, University of Ferrara, I-44121 Ferrara, Italy
}

Received August 2, 2018; Accepted January 22, 2019

DOI: $10.3892 /$ mco.2019.1826

\begin{abstract}
Recurrent differentiated thyroid carcinoma can easily be detected by means of ultrasound (US) and thyroglobulin, and often requires further surgical intervention. Revision surgery is often a technical challenge with significant risk of complications, considering the altered anatomy, with a possibility of leaving behind residual neoplasm. Preoperative US-guided tattooing localization has been introduced to reduce and prevent these potential problems during revision surgery. Encouraging results have been reported in the literature. Under US guidance, the lesion is identified and 0.5-2 $\mathrm{ml}$ of colloidal charcoal is injected in its proximity using a 23 gauge needle. The extraction is accompanied by injection at constant pressure of charcoal in order to leave a trace of pigment along the path of the needle till the skin. From April 2008 to January 2016 we performed revision surgery in 27 patients for lymph-nodes metastasis in differentiated thyroid cancer, using the technique of preoperative charcoal tattoo localization. Our previous study on the first group of 13 patients published in 2012, reported the preliminary results in terms of success rate and complications. The tolerance of charcoal injection was good for all patients and the procedure was demonstrated to be useful, contributing to the removal of metastatic lesion in $93 \%$ of procedures. We have registered minor surgical complica-
\end{abstract}

Correspondence to: Dr Andrea De Vito, Head and Neck Department, ENT and Oral Surgery Unit, Morgagni-Pierantoni Hospital, Forlì-and-Santa Maria delle Croci Hospital, Ravenna AUSL of Romagna, Via Forlanini 34, I-47121 Forlì, Italy

E-mail: dr.andrea.devito@gmail.com

Key words: charcoal tattoo, differentiated thyroid cancer recurrence, neck dissection, surgical complications, ultrasound tions during revision in the central compartment of the neck: Transitory hypoparathyroidism in 2 cases (11\%) and transitory vocal cord paresis in 3 cases $(16 \%)$. Based on these results, preoperative charcoal tattoo localization in revision surgery of the neck for differentiated thyroid cancer recurrence can be considered a safe technique, easy to perform, with low-costs and useful during surgical procedures, providing a significant reduction of iatrogenic damage and risks.

\section{Introduction}

5 to $40 \%$ of patients treated for differentiated thyroid cancer (DTC) can be affected by persistent or recurrent disease (1). Treatment with radioiodine alone, in a significant number of cases, is able to completely eliminate tumor recurrence. Nevertheless, up to $30 \%$ of tumors will not show ${ }^{131}$ I uptake. In these cases, a surgical management of the lesion is required (2). During revision surgery, a significant number of intraoperative complications can occur, especially in the central compartment of the neck. Alteration of the normal anatomy, fibrosis and scar tissue formation in the neck makes identification and preservation of the recurrent laryngeal nerves and parathyroid glands difficult (3). Another aspect to consider during revision surgery is the achievement of oncologic radicality. Recurrent laryngeal nerves and parathyroids may be encased in fibrotic tissue, making them indistinguishable from pathologic tissue. There is a considerable high risk of leaving residual neoplasm in the field (4).

In order to simplify localization and excision of neoplastic foci during revision surgery for DTC recurrence, we have employed a new technique of preoperative ultrasound-guided tattooing (US-tattoo) for the detection of the structures to be removed, likewise to the US-tattoo technique routinely applied in patients affected by breast cancer for more than 15 years (5). To date this procedure is supported by only four 4 studies reporting encouraging results $(2,3,6,7)$. The first 
13 patients have been reported in our previous article where the preliminary results have been reported in terms of success rate and complications (7).

In this study, we report our overall experience with the technique of fine-needle injection of charcoal pigment under US guidance for localization, and consequent surgical excision of DTC lymphatic metastasis in the neck.

\section{Materials and methods}

This was a retrospective analysis, performed in the Head and Neck Department, Ear Nose and Throat (ENT) Unit of Santa Maria delle Croci Hospital, Ravenna, Italy. All procedures performed in this study involving human participants were in accordance with the ethical standards of the institutional and/or national research committee, and with the 1964 Helsinki declaration and its later amendments or comparable ethical standards. The study was designed and conducted in compliance with the principles of Good Clincal Practice regulations and the Helsinki declaration. Written informed consent was obtained from patients before inclusion in this study.

From April 2008 to January 2016, a prospective study was conducted on patients who previously underwent surgical treatment for DTC, and thereafter presented to our centre with a suspected DTC recurrence in the neck. The initial operation and pTNM classification are shown in Table I. Vocal folds motility by videofiberoptic endoscopic assessment and parathyroid function was preoperatively verified. Radioiodine treatment was administered after surgical treatment in all patients population, except for one.

Recurrence or suspicious lesions discovered during follow-up, by means of US and TSH-stimulated Tg determination were investigated by cytology (FNA), in association with Tg determination in the washout of fine needle aspiration (FNAB-Tg), following the TI-RADS criteria (8). Recurrent lesions have been detected both in central and lateral compartment of the neck. In order to perform a super-selective neck dissection around the metastatic node, suspicious metastatic lymph-nodes in the lateral groups are also marked $(2,3,6)$. Informed consent was obtained from all patients.

A suspension of active charcoal $80 \mathrm{mg} / 2 \mathrm{ml}(4 \%)$, commonly used for tattooing breast cancer lesions, was used (composition: active charcoal $80 \mathrm{mg}+$ Polysorbate-80 $80 \mathrm{mg}+$ water for injectable preparations to reach $2 \mathrm{ml}$ ). Before aspiration and injection, the charcoal vial is heated by keeping it between the operator hands for a few minutes. This is done to prevent blockage of the needle tip by charcoal particles. The lesion is identified under US guidance; US imaging is performed using a machine with multi-frequency probe (7-12 MHz Esaote MyLab 70).

After identification, a 23-gauge needle is inserted near the suspected lesion, since we prefer to avoid injecting inside the lesion so as not to compromise histological examination. At this point we can proceed with injection of $0.5-2 \mathrm{ml}$ of charcoal by means of a $5 \mathrm{ml}$ syringe. The amount to be injected depends on the depth of the lesion. In proximity of the suspected neoplasm, a store of colouring which we define as charcoal 'puddle' takes form (Fig. 1). Extraction of the needle is accompanied by an injection at constant pressure of the charcoal in order to leave a trace of colouring along the way of the needle till the skin, where a tattoo resembling a little nevus will persist for some weeks. This last step provided a valid indication about the direction to follow during surgical operation, in order to reach the suspected metastatic lesion.

This procedure can be performed from just a few days to some weeks before surgery, as it has been demonstrated that charcoal remains in place for at least 3 months after injection $(9,10)$. Charcoal injection is performed in the Endocrinology unit as an ambulatory setting. The patient can return home after at least 20 min of observation. The patient is advised to rest, avoid in particular bending the neck and the head, and raising heavy loads after the procedure. In case of soreness or pain in the area of needle insertion, the use of a common analgesic is recommended. Only a descriptive analysis has been performed.

\section{Results}

A total number of 27 patients (20 females and 7 males) with an average age of 46 years (range, 25-73 years) presented to our centre ( 1 of them was initially treated in another hospital) with a suspected DTC recurrence in the neck (Table I). The average time of recurrence was 19 months (range, 3-84 months). Preoperative injection was well tolerated in all cases with patients complaining of the same mild discomfort of a FNA, experienced previously by all of them. No complications related to the procedure of US-tattooing were observed.

In the last 93 months (April 2008-January 2016) we have re-operated 27 patients with suspected metastatic lesion of DTC using the technique of US-tattoo localization. The primary cancer was always papillary carcinoma (with Warthin like aspects in 1 case, no. 22). Immediately prior to surgery in the operating room, we perform another US examination to have a better conception of the metastatic lesion and to define its anatomical relation. All the operations were performed by the same expert surgeon (FS and ADV). To recognize neural structures and assess their functionality we made use during surgery of Bovie Neuro-Pulse ${ }^{\mathrm{TM}}$ surgical nerve locator.

For the 16 cases the target was a suspected level VI node (nos. 2, 3, 5-8, 12-16, 18-21) (Table I; Fig. 2).

The first operation was a total thyroidectomy in 4 cases, thyroidectomy with dissection of level VI in 7 cases, and thyroidectomy with dissection of level VI in associated with selective neck dissection in 5 cases (Table I). The re-operation for these patients consisted of level VI dissection (comprehensive of the marked node) in 3 cases, revision of level VI dissection in 13 cases; number of nodes removed in central or lateral compartment is reported in Table I. For patients firstly not undergone central compartment lymphadenectomy, the use of charcoal tattoo is of great importance to assure inclusion of the suspected node in the dissection.

In patients already submitted to central compartment dissection, the operation consisted of removal of the charcoal-localized node possibly associated with fatty or connective tissue (suspected of containing other lymphatic nodes) surrounding the targeted lesion. In four patients, cytology was not suggestive of a definitive diagnosis. However, on the basis of US aspects and TG determination, we proceeded to perform revision surgery and metastatic lesion was found in 2 cases (nos. 12 and 16), in the other one a metastatic lesion 


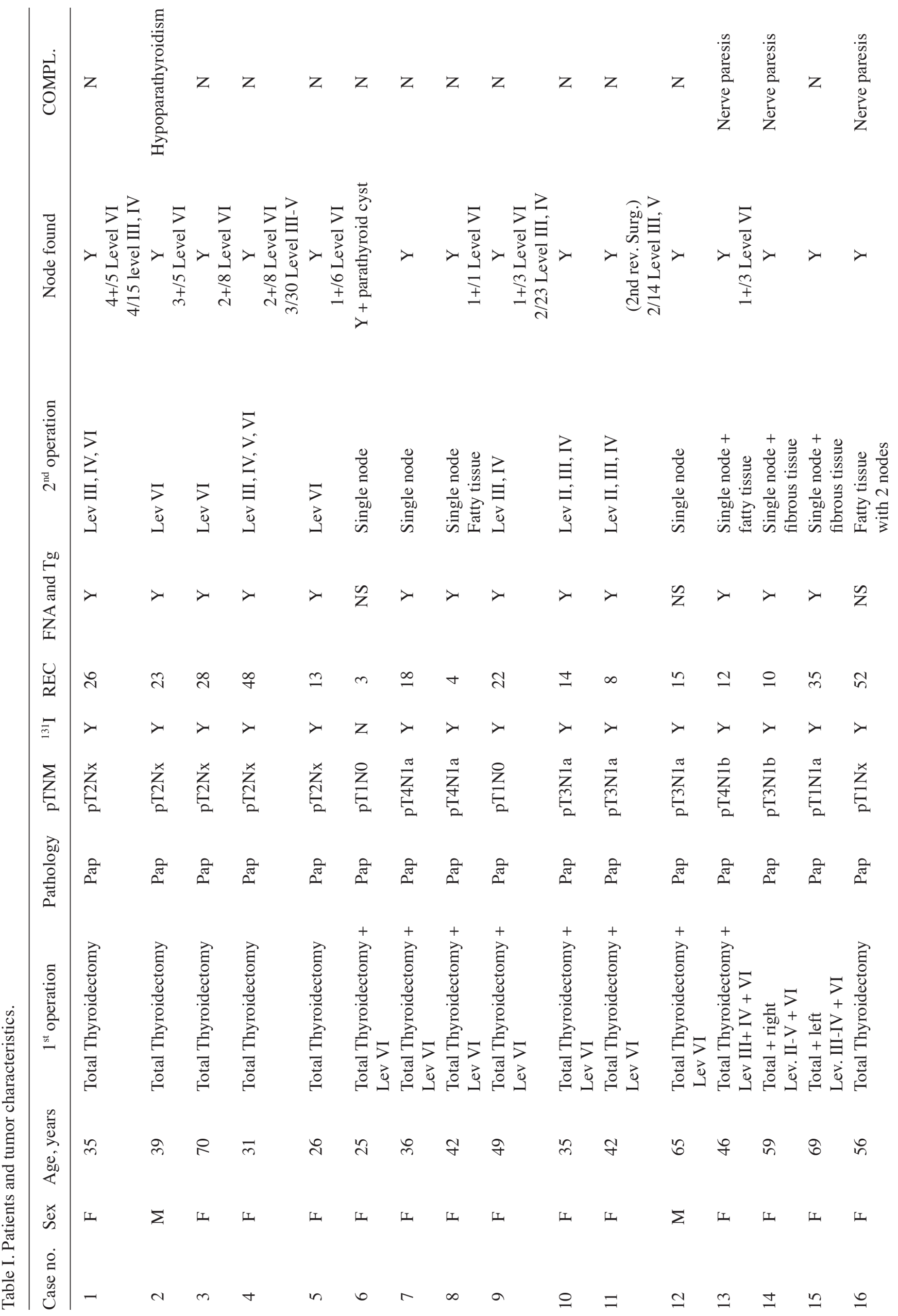




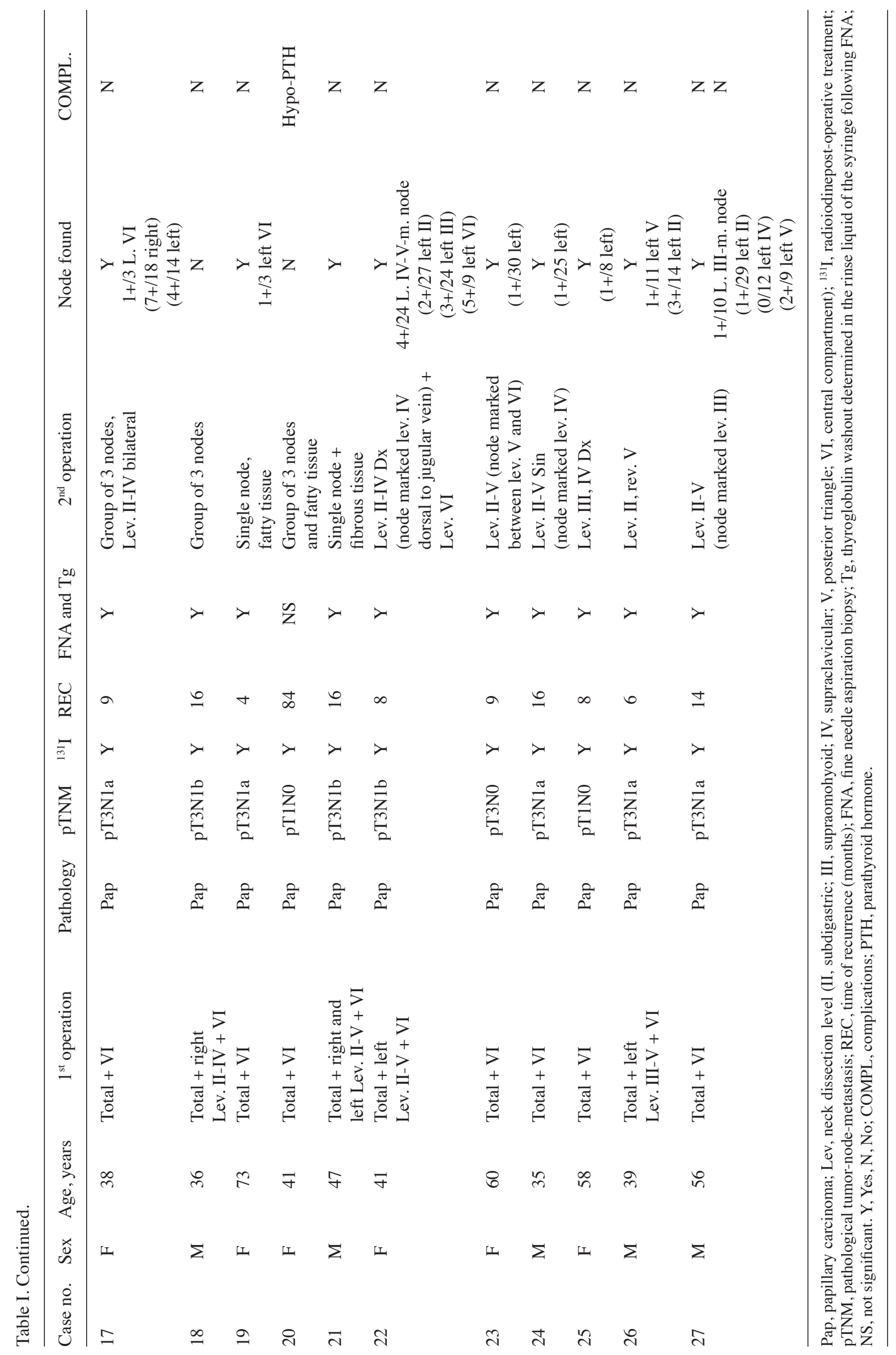




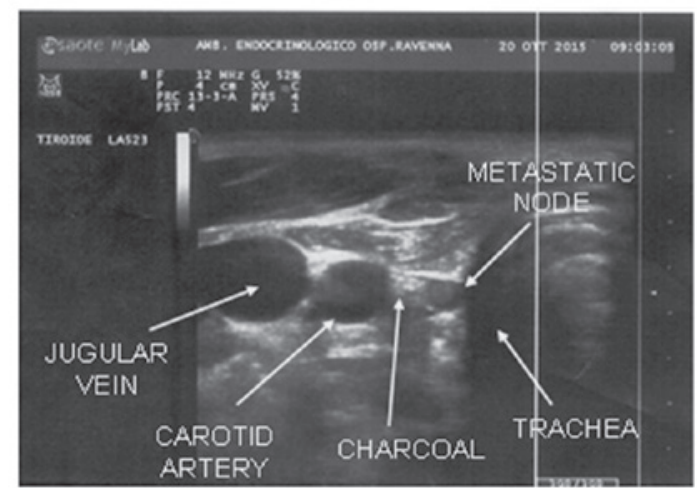

Figure 1. Ultrasound performed just before surgery showing the localization of the lymph node, anatomical associations and the accumulation of charcoal.

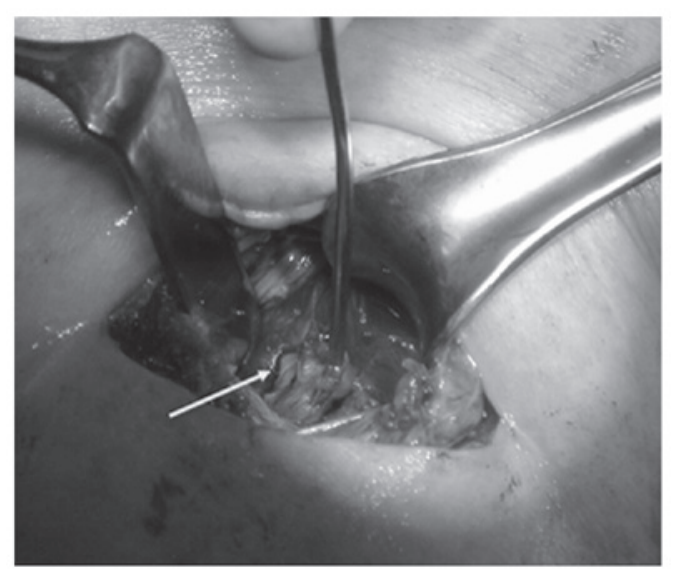

Figure 2. Traces of charcoal near a suspected node during area 6 revision surgery.

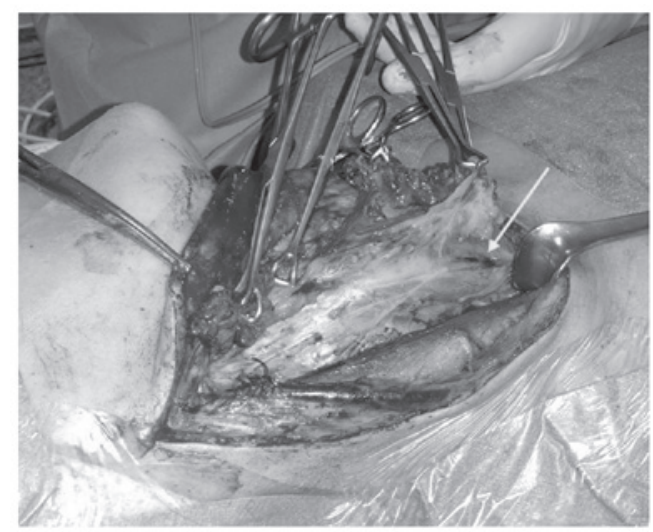

Figure 3. Accumulation of charcoal near the level IV node and in the previous needle path providing the direction to follow during dissection. The arrow indicates the charcoal trace created during needle extraction.

was found associated with parathyroid cyst (no. 6) and finally the suspected lesion, which is of small dimension ( $3 \times 5 \mathrm{~mm})$, were not confirmed at histological examination, even if a group of 3 nodes were removed (no. 20).

The last was the unique case in our experience we did not observe any trace of charcoal in proximity of the suspected lesion during the surgical operation. We found colouring traces just in the first layer of dissection (skin, subcutaneous and scar tissue), which is of poor usefulness for our surgical aim. Similar to what occurred in case no. 20 , in patient no. 18 the metastatic node was not found despite the removal of three nodes around the coal deposit (Table I). For these patients, considering the small size of the lesion together with objective surgical difficulties, we decided for a further radioiodine treatment followed by close controls and reoperation in case of volumetric increase of the lesion.

After revision surgery in the central compartment, we observed minor complications in 5 cases: 2 patients suffered temporary hypoparathyroidism (solved with medical therapy) and 3 experienced temporary recurrent nerve paralysis. In a subset of 9 patients (patients nos. 1,9-11 and 23-27) we marked exclusively lesions in lateral compartment of the neck. In one case (no. 26) 2 distinct marks were performed and in 1 of these, in the level II area, we observed a widespread diffusion of charcoal. In all other cases we could follow the trace created during extraction of the needle, and found the charcoal injected quite localized on the suspected lesion and therefore useful for surgical manoeuvres (Fig. 3).

The histological examination has given a favourable outcome, confirming the removal of metastatic lesion in all cases. No complications related to lateral neck dissections were registered.

In case no. 4 and no. 22, we performed a double marking of a lesion localized in VI area and another respectively in level III and IV (dorsal to jugular vein). Removal of metastatic lesion was obtained in both cases with no registration of surgical complications. Considering overall results, metastatic lesions were found in 25 out of 27 patients (92\%) (Table I). Follow up with US and Tg determination of at least 6 months confirmed the positive outcome of surgery.

\section{Discussion}

DTC is the most common endocrine tumour. Despite the increase in the number of cases in recent years, its course is favourable with a survival rate of 10 years till $90 \%$, and its incidence in all deaths for neoplastic diseases is at only $0,5 \%$ (11). Lymphatic metastasis of DTC has been considered, until a short time ago of negligible importance for the prognosis. Various studies have reported percentages of survival rates of 10 years till $95 \%$ for papillary carcinoma, and till $70 \%$ for the follicular variant (12-16). Recently, however, major attention is placed on the concept of 'disease free survival' considering, in spite of high values of survival, the not negligible incidences of disease recurrence (15-35\% also to 20 years), with a meaningful impact on the quality of life (due to the necessity of surgical reoperations and/or of elevated doses of radioiodine) $(17,18)$.

The incidence of DTC recurrence after thyroidectomy has increased with the routine employment of ultrasound (US) and thyroglobulin (Tg). Revision surgery in the neck for recurrent/persistent DTC is associated with increased morbidity compared with primary surgery because of the presence of scar tissue and disruption of the normal fascial planes and anatomy. This may result in a greater risk of injury to nerves and other important anatomical structures (19). These concepts are valid especially for area VI surgical revision. In spite of every therapeutic attitude regarding the N0 necks in DTC, re-operations 
in the central compartment may be necessary, even when it has been performed during the first operation. In fact, radicality is very difficult to obtain, especially for nodes situated on the superior edge of mediastinal space. Risk of damaging laryngeal nerves can reach $20 \%$ (ranging from 0.7 to $4.5 \%$ during first operation) and risk of hypoparathyroidism can increase to $30 \%$ (ranging from 8 to $13 \%$ in primary procedures) $(20,21)$.

Even though our study consists of a small number of treated patients, we observed a transitory hypoparathyroidism in 2 cases (11\% if we consider only 18 cases of area 6 revision) and a transitory vocal cord paresis in 3 (16\% referring exclusively to area 6 revision). No damages of important anatomical structures were registered during lateral neck dissection. Greater morbidity during central compartment revision is related to difficulties in identification and preservation of recurrent laryngeal nerves and parathyroid glands. To prevent recurrent nerve injuries, it is possible to make use of intraoperative neurological monitoring and/or rely on some surgical tricks such as identification of each nerve low in the tracheoesophageal groove, distant from the thyroid bed and the use of meticulous surgical dissection from inferior to superior (4). In order to preserve the function of the parathyroid glands, devascularisation should be prevented. The inferior thyroid artery should be respected (4).

The gold standard for revision surgery should be a reliable procedure to guide revision surgery. To date the most common intraoperative procedure is the use of a gamma probe. Efficacy of this technique however can be limited by false negative and false positive findings. It is only useful in cases of radioiodine-avid foci (22-24). Other modalities such as intraoperative US exploration hook needle insertion or tattooing using blue dye have also been described. However, their efficacy, safety and feasibility are not well demonstrated (25-27). Other radio guided methods as well as other radiotracers such us FDG were recently developed for the intraoperative guidance of non-radioiodine avid cancer, but cost, availability and signal-to-noise issues may limit their widespread use (28-30).

Despite all these procedures, revision surgery of central compartment dissection remains difficult even in expert hands. Given this context, the US-tattoo localization technique can represent an instrument of considerable usefulness $(2,3,6,7)$.

Active charcoal for its physical and pharmacological properties, which ensure limited diffusion and good stability, is the ideal substance for marking of target in soft tissues. In our experience this technique has been of significant utility in identifying metastatic lesions during revision surgery in a great majority of cases (93\%). In a total of 30 procedures, we registered only in 1 case an unexplained absence of coal and in a further one, an excessive widespread in the surgical field. Furthermore, this technique, executable even several week before surgery, is easy to implement and at a very low cost. Finally, another aspect of great importance is the good tolerability and the complete absence of complications related to the procedure.

In conclusion, based on our experience we can suggest that US-tattoo localization of lymphatic neck metastasis of DTC is a safe technique, of low cost and extremely useful in facilitating surgical procedures, especially in difficult revision surgery of the neck. This technique allows risk reduction of iatrogenic complications, especially of the parathyroid glands and recurrent laryngeal nerves during area VI revision surgery.

\section{Acknowledgements}

Not applicable.

\section{Funding}

No funding was received.

\section{Availability of data and materials}

The datasets used and/or analyzed during the current study are available from the corresponding author on reasonable request.

\section{Authors' contributions}

FS and ADV conceived and designed the study, acquired, analysed and interpreted the data, revised the manuscript, gave final approval of the version to be published and agreed to be accountable for all aspects of the work. FB, GM, SSR, CC, FR, CV and MP conceived and designed the study, drafted the manuscript, analysed the data, gave final approval of the version to be published and agreed to be accountable for all aspects of the work

\section{Ethics approval and consent to participate}

Due to the retrospective nature of the present study, the requirement for ethical approval was waived. Written informed consent was obtained from all individual participants included in the study.

\section{Patient consent for publication}

Written informed consent was obtained from all individual participants included in the study.

\section{Competing interests}

The authors declare that they have no competing interests.

\section{References}

1. Schlumberger MJ: Papillary and follicular thyroid carcinoma. N Engl J Med 338: 297-306, 1998.

2. Hartl DM, Chami L, Al Ghuzlan A, Leboulleux S, Baudin E, Schlumberger M and Travagli JP: Charcoal suspension tattoo localization for differentiated thyroid cancer recurrence. Ann Surg Oncol 16: 2602-2608, 2009.

3. Kang TW, Shin JH, Han BK, Ko EY, Kang SS, Hahn SY, Kim JS and Oh YL: Preoperative ultrasound-guided tattooing localization of recurrences after thyroidectomy: Safety and effectiveness. Ann Surg Oncol 16: 1655-1659, 2009.

4. Kim MK, Mandel SH, Baloch Z, Livolsi VA, Langer JE, Didonato L, Fish S and Weber RS: Morbidity following central compartment reoperation for recurrent or persistent thyroid cancer. Arch Otolaryngol Head Neck Surg 130: 1214-1216, 2004.

5. Mathieu MC, Bonhomme-Faivre L, Rouzier R, Seiller M, Barreau-Pouhaer L and Travagli JP: Tattooing breast cancers treated with neoadjuvant chemotherapy. Ann Surg Oncol 14: 2233-2238, 2007. 
6. Chami L, Hartl D, Leboulleux S, Baudin E, Lumbroso J, Schlumberger M and Travagli JP: Preoperative localization of neck recurrences from thyroid cancer: Charcoal tattooing under ultrasound guidance. Thyroid 25: 341-346, 2015.

7. Soprani F, Bondi F, Puccetti M and Armaroli V: Charcoal tattoo localization for differentiated thyroid cancer recurrence in the central compartment of the neck. Acta Otorhinolaryngol Ital 32: 87-92, 2012.

8. Wang Y, Lei K-R, He Y-P, Li XL, Ren WW, Zhao CK, Bo XW, Wang D, Sun CY and Xu HX: Malignancy risk stratification of thyroid nodules: Comparisons of four ultrasound Thyroid Imaging Reporting and Data Systems in surgically resected nodules. Sci Rep 7: 11560, 2017.

9. Bonhomme-Faivre L, Depraetere P, Savelli MP, Amdidouche D, Bizi E, Seiller M and Orbach-Arbouys S: Charcoal suspension for tumor labelling modifies macrophage activity in mice. Life Sci 66: 817-827, 2000

10. Biffoni M, Scipioni P and Macrina N: Surgical treatment of differentiated thyroid cancer recurrence. L'Endocrinologo 10 : 143-148, 2009.

11. Moley JF and Wells SA: Compartment-mediated dissection for papillary thyroid cancer. Langenbecks Arch Surg 384: 9-15, 1999.

12. Lundgren CI, Hall P, Dickman PW and Zedenius J: Clinically significant prognostic factors for differentiated thyroid carcinoma: A population-based, nested case-control study. Cancer 106: 524-531, 2006.

13. Shaha A: Treatment of thyroid cancer based on risk groups J Surg Oncol 94: 683-691, 2006

14. Kupferman ME, Patterson M, Mandel SJ, LiVolsi V and Weber RS: Patterns of lateral neck metastasis in papillary thyroid carcinoma. Arch Otolaryngol Head Neck Surg 130: 857-860, 2004.

15. Hassanain $\mathbf{M}$ and Wexler $\mathbf{M}$ : Conservative management of well-differentiated thyroid cancer. Can J Surg 53: 109-118, 2010

16. Shaha AR, Shah JP and Loree TR: Patterns of nodal and distant metastasis based on histologic varieties in differentiated carcinoma of the thyroid. Am J Surg 172: 692-694, 1996.

17. Hay ID, Bergstralh EJ, Grant CS, McIver B, Thompson GB, van Heerden JA and Goellner JR: Impact of primary surgery on outcome in 300 patients with pathologic tumor-node-metastasis stage III papillary thyroid carcinoma treated at one institution from 1940 through 1989. Surgery 126: 1173-1181, discussion $1181-1182,1999$.

18. Pai SI and Tufano RP: Reoperation for recurrent/persistent well-differentiated thyroid cancer. Otolaryngol Clin North Am 43: 353-363, ix, 2010

19. Betka J, Mrzena L, Astl J, Nemec J, Vlcek P, Taudy M and Skrivan J: Surgical treatment strategy for thyroid gland carcinoma nodal metastases. Eur Arch Otorhinolaryngol 254 (Suppl 1): S169-S174, 1997.
20. Scheumann GF, Seeliger H, Musholt TJ, Gimm O, Wegener G, Dralle H, Hundeshagen $\mathrm{H}$ and Pichlmayr R: Completion thyroidectomy in 131 patients with differentiated thyroid carcinoma. Eur J Surg 162: 677-684, 1996.

21. Travagli JP, Cailleux AF, Ricard M, Baudin E, Caillou B, Parmentier C and Schlumberger M: Combination of radioiodine (131I) and probe-guided surgery for persistent or recurrent thyroid carcinoma. J Clin Endocrinol Metab 83: 2675-2680, 1998.

22. Rubello D, Salvatori M, Ardito G, Mariani G, Al-Nahhas A, Gross MD, Muzzio PC and Pelizzo MR: Iodine-131 radio-guided surgery in differentiated thyroid cancer: Outcome on 31 patients and review of the literature. Biomed Pharmacother 61: 477-481, 2007.

23. Salvatori M, Ardito G, Pelizzo MR, Mariani G, Gross M, Al-Nahhas A and Rubello D: Treatment of local and regional recurrences of differentiated thyroid cancer by radio-guided surgery with iodine-131. Nucl Med Rev Cent East Eur 9: 119-124, 2006.

24. Lucchini R, Puxeddu E, Calzolari F, Burzelli F, Monacelli M, D'Ajello F, Macaluso R, Giammartino C, Ragusa M, De Feo P, et al: Recurrences of thyroid well differentiated cancer: Ultrasonography-guided surgical treatment. Minerva Chir 63: 257-260, 2008

25. Duprez R, Lebas P, Marc OS, Mongeois E, Emy P and Michenet P: Preoperative US-guided hook-needle insertion in recurrent lymph nodes of papillary thyroid cancer: A help for the surgeon. Eur J Radiol 73: 40-42, 2010.

26. Sippel RS, Elaraj DM, Poder L, Duh QY, Kebebew E and Clark OH: Localization of recurrent thyroid cancer using intraoperative ultrasound-guided dye injection. World J Surg 33: 434-439, 2009

27. Gulec SA, Eckert M and Woltering EA: Gamma probe-guided lymph node dissection ('gamma picking') in differentiated thyroid carcinoma. Clin Nucl Med 27: 859-861, 2002.

28. Grant CS, Thompson GB, Farley DR, Richards ML, Mullan BP and Hay ID: The value of positron emission tomography in the surgical management of recurrent papillary thyroid carcinoma. World J Surg 32: 708-715, 2008.

29. Borsò E, Grosso M, Boni G, Manca G, Bianchi P, Puccini M, Arganini M, Cabria M, Piccardo A, Arlandini A, et al: Radioguided occult lesion localization of cervical recurrences from differentiated thyroid cancer: Technical feasibility and clinical results. Q J Nucl Med Mol Imaging 57: 401-411, 2013.

30. Molina MA, Goodwin WJ, Moffat FL, Serafini AN, Sfakianakis GN and Avisar E: Intra-operative use of PET probe for localization of FDG avid lesions. Cancer Imaging 9: 59-62, 2009. 